\title{
The Effect of KRAS and NRAS Variants on the Prognosis of Colorectal Cancer among the Western Saudi Population
}

\author{
Rami Nassir ${ }^{1 *}$, Ghada Esheba1,2 \\ ${ }^{1}$ Department of Pathology, School of Medicine, Umm Al-Qura University, Makkah, Saudi Arabia \\ ${ }^{2}$ Department of Pathology, Faculty of Medicine, Tanta University, Tanta, Egypt \\ Email: ^rmnassir@uqu.edu.sa
}

How to cite this paper: Nassir, R. and Esheba, G. (2021) The Effect of KRAS and NRAS Variants on the Prognosis of Colorectal Cancer among the Western Saudi Population. Journal of Biosciences and Medicines, 9, 81-93.

https://doi.org/10.4236/jbm.2021.96008

Received: May 9, 2021

Accepted: June 15, 2021

Published: June 18, 2021

Copyright $\odot 2021$ by author(s) and Scientific Research Publishing Inc. This work is licensed under the Creative Commons Attribution International License (CC BY 4.0).

http://creativecommons.org/licenses/by/4.0/

\begin{abstract}
Colorectal Cancer (CRC) is one of the most common cancer with high mortality rate globally and the second leading cancer in Saudi Arabia. RAS oncogenes play critical roles in the regulation of the cellular function and any mutation in these genes leads to develop CRC. Therefore, we identified the most common mutations in KRAS and NRAS genes by applying next generation sequencing (NGS) then, we assessed the correlation between these mutations and the clinicopathological features. KRAS-mutated carcinoma was significantly associated in patients who were older than 60 years old $(83.3 \%$ vs $16.7 \%, \mathrm{P}=0.039)$ and it was associated with female patients as well, $(83.3 \%$ vs $30 \%, \mathrm{P}=0.039$ ). Also, KRAS-mutated carcinoma was significantly associated with mucinous differentiation $(85.7 \%$ vs $14.3 \% ; \mathrm{P}=0.012)$ and higher tumor grade $(\mathrm{P}=0.014)$. In addition, the number of KRAS mutations per case was significantly associated with depth of the invasion $(\mathrm{p}=0.049)$. The most common mutation was a missense mutation and it was highly associated with age and gender (both, $\mathrm{p}=0.039$ ). Also, it was highly associated with tumor grade and with mucinous differentiation $(\mathrm{p}=0.014, \mathrm{p}=0.012)$, respectively. On the other hand, NRAS mutated carcinoma was associated only with distant metastasis however, this association was not significant $(\mathrm{p}=0.064)$. For overall survival, KRAS-mutated carcinomas had a significantly worse overall survival $(\mathrm{p}=0.025)$. While, no significant association was between NRAS mutation and overall survival $(\mathrm{p}=0.985)$. We believe that KRAS and NRAS genes can be prognostic factors for CRC patients and the information obtained may contribute for better diagnosis and therapeutic effect.
\end{abstract}

\section{Keywords}

Colorectal Cancer, KRAS, NRAS 


\section{Introduction}

Colorectal Cancer (CRC) is the second leading cancer type of the estimated number of new cancer cases in 2016 in Saudi Arabis (12.6\%) (Saudi Cancer registry, 2016) for both genders. According to the American Cancer Society, CRC remains one of the main causes of high rate of cancer related death in the world, regardless of the significant improvement in the prevention, screening and therapy. CRC arises due to the transformation of the normal colonic epithelium as a result of the alteration in the genetics and the epigenetics of these cells which promotes the tumor growth. The progression of the molecular level in the tumor cells will cause genetic instability and genetics defect which leads to the development of sporadic CRC. The incidence rate and the mortality of CRC differ based in etiological factors such as age, gender, genetic background and environmental factors [1] [2] [3] [4].

Approximately, $60 \%$ of CRC caries mutations in the RAS oncogene, either KRAS, NRAS or both. KRAS and NRAS play an important role in regulating many cellular functions including cellular proliferation, apoptosis, migrations and differentiation [5] [6]. The most commonly reported mutation in the KRAS gene is located on exon 2 and consequently changes the codons 12 and codon 13 . Also, another frequently reported mutation in codon 16 and 146 have been reported, however, it has lower effect on the development of the tumor cells. On the other hand, the most commonly reported mutation in the NRAS gene is located in codon 61 and 12 [7] [8]. Activating mutations in the KRAS gene has been found to be predictor of response to EGFR-targeted therapies, therefore, examining the KRAS and NRAS mutations are an essential step in clinical diagnosis and proper treatment.

Most tumors are formed from clones that carry different mutations which are called Tumor Heterogeneity. This heterogeneity has significant impact on cancer development and the response to a treatment. Since next generation sequencing becomes applicable, these genetic variations or tumor heterogeneity, can be clearly identified. Also, it can reveal any co-existing mutations in different genes [6] [9]. While defining the risk mutations and treatment approach, much needs to be done to correlate these mutations with the clinicopathological outcomes. There are discrepancies in the literature regarding prognosis of KRAS mutation in colorectal cancer patients and the reports are contradictory.

To the best of our knowledge, no data has been published before concerning the effect of KRAS and NRAS mutations and their correlations with the clinicopathological variables and overall survival in Saudi patients with colon cancer.

Therefore, and to address the major gap in our knowledge of the different roles of these genetic variations and how they correlate with the pathological findings clinically, we aimed to identify the risk molecular variants and correlate them with the clinicopathological characteristics in Saudi patients with CRC. In addition, we assessed the role of these correlations in determining the prognosis, treatment personalization and overall survival. 


\section{Material and Methods}

\subsection{Patients and Tissue Samples}

The study included clinical and genotyped data for 16 patients diagnosed with colorectal cancer form local hospital in the western region of Saudi Arabia between 2013 and 2016. This study was approved and conducted under the ethics regulation by the ethical committee at the school of medicine at Umm Al-Qura University. All tissue sections were evaluated after staining using hematoxylin/eosin (H\&E) by a histopathologist. The inclusion criteria were: surgical colectomy cases that were diagnosed as colon cancer provided that the clinical data such as age, gender, tumor size, lymph node status and stage were present. Cases with no available data were excluded from this study. Furthermore, any patient who had undergone chemotherapy prior to surgery was also excluded from the study. Evaluation of the histopathological features was performed. The histological parameters assessed were tumor size, tumor grade, histological subtype, mucinous histology, depth of invasion (pT), lymph node involvement/metastasis $(\mathrm{pN})$, and lymphovascular invasions.

\subsection{Tumor Dissection and DNA Extraction}

After evaluating the cases who were eligible for the current study, we included 16 cases qualified for molecular analysis. From each selected case, 10-nm thick tissue section obtained from paraffin-embedded samples was collected in the Eppendorf safe-lock tube to be prepared for DNA extraction. From each selected case, 10-nm-thick tissue sections obtained from paraffin-embedded samples were collected in the Eppendorf safe-lock tube to be prepared for DNA extraction. The DNA was extracted from the tissue collected using the QIAamp DNA FFPE Tissue C-KIT (Qiagen, Hilden, Germany) according to the protocol of the manufacture. The extracted DNA was eluted into $40 \mathrm{~nL}$ of buffer, quantified using two methods: NanoDrop microvolume sample retention system (Thermo Fisher Scientific NanoDrop Products, Hanover Park, IL, USA). The second method of quantification was using Qubit, DS (Thermo Fisher Scientific, Waltham, MA, USA) and stored at $4^{\circ} \mathrm{C}$. All the 16 cases were fulfilled the recommended DNA quality required for the molecular analysis.

\subsection{Next Generation Sequencing}

For all 16 cases, 20ng of DNA extracted was prepared for sequencing. The assessment of 15 genes (AKT1, GNA11, NRAS, BRAF, GNAQ, PDGFRA, EGFR, c-KIT, PIK3CA, ERBB2, KRAS, RET, FOXL2, c-MET and TP53) was applied using TruSight Tumor 15 (Illumina, San Diego, CA, USA). The resulting pooled libraries were quality controlled using The Qubit ${ }^{\circledR}$ dsDNA HS (High Sensitivity) Assay c-KIT make DNA quantitation easy and accurate. Sequencing was performed with paired-end reads on the MiSeq Platform (Illumina ${ }^{\circledR}$ ). 


\subsection{Detection of Variations}

The sequenced reads were arranged and compared to the hg19/GRCh37 reference sequence and analyzed using the MiSeq reporter (Illumina). To identify variants in breast tissue samples, BaseSpcae Variant Interpreter (Illumina) was applied. The called variants were considered somatic malignant tumors of the breast (SNOMEDCT) version 4.0.7.6. To reduce the false-positive rate, we set the cutoff value as follows: read depth $>100$, Indel repeat length $<8$; allele frequency of mutant reads $>1 \%$, genotype quality $>30$.

\subsection{Statistical Analysis}

Association between KRAS and NRAS gene polymorphism and the clinicopathological parameters assessed by applying Chi-square statistical test and Pearson correlation. Survival analyses were estimated using the Kaplan-Meier plot method and compared using the log-rank test. Cox proportional hazards model was used to estimate the hazard ratio (HR) of each clinicopathological variables for OS and DFS. All predictors with P-value $<0.05$ in univariate Cox analyses were used in multivariate analysis. P-values were 2 -tailed and considered significant when $<0.05$. Statistical analyses were carried out using IBM SPSS statistics 22.0 software (IBM Corp., Armonk, NY, USA).

\section{Results}

\subsection{Patients Characteristics}

The details of clinicopathological features of the colon cancer cases who are included in the present study are summarized in Table 1. Briefly, the mean age was $61.37 \pm 12.96$ years (range $41-87$ years). Three patients (18.8\%) developed metastasis and four patients (25\%) died by the end of the follow up. Five patients (31.3\%) had positive lymph nodes. Ten cases $(62.5 \%)$ were in the right colon, while the remaining 6 cases (37.5\%) were in the left colon/rectum.

\subsection{Mutation Profiles}

While KRAS and NRAS mutations were identified 8/16 (50\%) and 4/16 (25\%) respectively of the tumor, all the patients in this study group had mutation in p53 gene (100\%). Moreover, 5 patients were found to carry multiple mutations, including patients with co-existence of $K R A S$ and/or $N R A S$ genes mutations. One patient had a mutation in $E G F R$ gene and another patient had a mutation in the PIK3CA gene. One of the patients had multiple mutations in the following genes: $A K T 1, N R A S, P D G F R A, E G F R, \mathrm{c}-K I T, E R B B 2, \mathrm{c}-M E T$ and $T P 53$.

\subsection{Correlation of KRAS and NRAS Mutations with Clinicopathological Variables}

The frequency and the association of clinicopathological features of patients who harbored the mutant KRAS and NRAS tumors are shown in Table 2.

KRAS-mutated carcinomas were significantly more common in patients who 
are older than 60 years $(83.3 \%$ vs $16.7 \%, \mathrm{P}=0.039)$. Female patients as well, were found to be more likely to have KRAS mutation than male patients $(83.3 \%$ vs $30 \%, \mathrm{P}=0.039)$. Also, KRAS-mutated carcinomas exhibited more mucinous differentiation $(85.7 \%$ vs $14.3 \%$; $\mathrm{P}=0.012)$. Furthermore, KRAS-mutated carcinomas were significantly associated with higher tumor grade $(\mathrm{P}=0.014)$. On the other hand, age, gender, location of the tumor, mucinous differentiation, depth

Table 1. Clinicopathological features of colorectal cancer patients included in the study.

\begin{tabular}{|c|c|c|c|}
\hline & & $\mathbf{N}$ & $\%$ \\
\hline \multirow{3}{*}{ Age } & Less than 60 & 6 & $37.5 \%$ \\
\hline & & & \\
\hline & More than 60 & 10 & $62.5 \%$ \\
\hline \multirow{3}{*}{ Gender } & Male & 10 & $62.5 \%$ \\
\hline & & & \\
\hline & Female & 6 & $37.5 \%$ \\
\hline \multirow{3}{*}{ Tumor Size } & Less than 5 & 7 & $43.8 \%$ \\
\hline & & & \\
\hline & More than 5 & 9 & $56.3 \%$ \\
\hline \multirow{3}{*}{ Tumor Site } & Proximal Colon & 11 & $68.8 \%$ \\
\hline & & & \\
\hline & Distal/Rectum & 5 & $31.3 \%$ \\
\hline \multirow{3}{*}{ Tumor Grade } & Well Differentiated & 3 & $18.8 \%$ \\
\hline & Moderately Differentiated & 8 & $50.0 \%$ \\
\hline & Poorly Differentiated & 5 & $31.3 \%$ \\
\hline \multirow{3}{*}{ Depth of Invasion (pT) } & $\mathrm{T} 1-\mathrm{T} 2$ & 7 & $43.8 \%$ \\
\hline & & & \\
\hline & $\mathrm{T} 3-\mathrm{T} 4$ & 9 & $56.3 \%$ \\
\hline \multirow{2}{*}{ Lymph Node Involvement (nT) } & Negative & 11 & $68.8 \%$ \\
\hline & Positive & 5 & $31.3 \%$ \\
\hline \multirow{2}{*}{ Lymph Node Metastasis (pM) } & No Metastasis & 13 & $81.3 \%$ \\
\hline & Metastasis & 3 & $18.8 \%$ \\
\hline \multirow{3}{*}{ Distant Detastasis } & No metastasis & 13 & $81.3 \%$ \\
\hline & & & \\
\hline & metastasis & 3 & $18.8 \%$ \\
\hline \multirow{2}{*}{ TNM } & Stage 1 - 2 & 8 & $50.0 \%$ \\
\hline & Stage $3-4$ & 8 & $50.0 \%$ \\
\hline \multirow{2}{*}{ Mucinous Histology } & No & 9 & $56.3 \%$ \\
\hline & Yes & 7 & $43.8 \%$ \\
\hline \multirow{2}{*}{$\mathrm{LVI}^{*}$} & Absent & 13 & $81.3 \%$ \\
\hline & Present & 3 & $18.8 \%$ \\
\hline \multirow[b]{2}{*}{ KRAS } & Negative & 8 & $50.0 \%$ \\
\hline & Positive & 8 & $50.0 \%$ \\
\hline \multirow[b]{2}{*}{ NRAS } & No Mutation & 12 & $75.0 \%$ \\
\hline & Mutation & 4 & $25.0 \%$ \\
\hline
\end{tabular}

${ }^{*}$ LVI: lymphovascular invasion. 
Table 2. The association between KRAS and NRAS mutations and the clinicopathological features.

\begin{tabular}{|c|c|c|c|c|c|c|c|c|c|c|c|}
\hline & & \multicolumn{4}{|c|}{ KRAS } & \multicolumn{6}{|c|}{ NRAS } \\
\hline & & \multicolumn{2}{|c|}{ No mutation } & \multicolumn{2}{|c|}{ Mutation } & \multirow{2}{*}{ P-value } & \multicolumn{2}{|c|}{ No Mutation } & \multicolumn{2}{|c|}{ Mutation } & \multirow{2}{*}{ P-value } \\
\hline & & $\mathbf{N}$ & $\%$ & $\mathbf{N}$ & $\%$ & & $\mathbf{N}$ & $\%$ & $\mathrm{~N}$ & $\%$ & \\
\hline \multirow[b]{2}{*}{ Age } & Less than 60 & 5 & $83.3 \%$ & 1 & $16.7 \%$ & \multirow{2}{*}{0.039} & 4 & $66.7 \%$ & 2 & $33.3 \%$ & \multirow{2}{*}{0.551} \\
\hline & More than 60 & 3 & $30.0 \%$ & 7 & $70.0 \%$ & & 8 & $80.0 \%$ & 2 & $20.0 \%$ & \\
\hline \multirow{2}{*}{ Gender } & Male & 7 & $70.0 \%$ & 3 & $30.0 \%$ & \multirow{2}{*}{0.039} & 7 & $70.0 \%$ & 3 & $30.0 \%$ & \multirow{2}{*}{0.551} \\
\hline & Female & 1 & $16.7 \%$ & 5 & $83.3 \%$ & & 5 & $83.3 \%$ & 1 & $16.7 \%$ & \\
\hline \multirow{2}{*}{ Tumor Size } & Less than 5 & 2 & $28.6 \%$ & 5 & $71.4 \%$ & \multirow{2}{*}{0.614} & 7 & $100.0 \%$ & 0 & $0.0 \%$ & \multirow{2}{*}{0.383} \\
\hline & More than 5 & 6 & $66.7 \%$ & 3 & $33.3 \%$ & & 5 & $55.6 \%$ & 4 & $44.4 \%$ & \\
\hline \multirow[b]{2}{*}{ Tumor Site } & Proximal Colon & 4 & $36.4 \%$ & 7 & $63.6 \%$ & \multirow[b]{2}{*}{0.106} & 8 & $72.7 \%$ & 3 & $27.3 \%$ & \multirow[b]{2}{*}{0.755} \\
\hline & Distal/Rectum & 4 & $80.0 \%$ & 1 & $20.0 \%$ & & 4 & $80.0 \%$ & 1 & $20.0 \%$ & \\
\hline \multirow{3}{*}{ Tumor Grade } & Well Differentiated & 3 & $100.0 \%$ & 0 & $0.0 \%$ & & 1 & $33.3 \%$ & 2 & $66.7 \%$ & \multirow{3}{*}{0.173} \\
\hline & $\begin{array}{c}\text { Moderately } \\
\text { Differentiated }\end{array}$ & 5 & $62.5 \%$ & 3 & $37.5 \%$ & 0.014 & 7 & $87.5 \%$ & 1 & $12.5 \%$ & \\
\hline & Poorly Differentiated & 0 & $0.0 \%$ & 5 & $100.0 \%$ & & 4 & $80.0 \%$ & 1 & $20.0 \%$ & \\
\hline \multirow{2}{*}{ Depth of Invasion (pT) } & $\mathrm{T} 1-\mathrm{T} 2$ & 4 & $57.1 \%$ & 3 & $42.9 \%$ & \multirow{2}{*}{0.614} & 6 & $85.7 \%$ & 1 & $14.3 \%$ & \multirow{2}{*}{0.383} \\
\hline & $\mathrm{T} 3-\mathrm{T} 4$ & 4 & $44.4 \%$ & 5 & $55.6 \%$ & & 6 & $66.7 \%$ & 3 & $33.3 \%$ & \\
\hline \multirow{2}{*}{$\begin{array}{c}\text { Lymph Node } \\
\text { Involvement (nT) }\end{array}$} & Negative & 7 & $63.6 \%$ & 4 & $36.4 \%$ & \multirow{2}{*}{0.106} & 8 & $72.7 \%$ & 3 & $27.3 \%$ & \multirow{2}{*}{0.755} \\
\hline & Positive & 1 & $20.0 \%$ & 4 & $80.0 \%$ & & 4 & $80.0 \%$ & 1 & $20.0 \%$ & \\
\hline \multirow{2}{*}{$\begin{array}{c}\text { Distant } \\
\text { Metastasis (pM) }\end{array}$} & No metastasis & 6 & $46.2 \%$ & 7 & $53.8 \%$ & \multirow{2}{*}{0.522} & 11 & $84.6 \%$ & 2 & $15.4 \%$ & \\
\hline & Metastasis & 2 & $66.7 \%$ & 1 & $33.3 \%$ & & 1 & $33.3 \%$ & 2 & $66.7 \%$ & 0.004 \\
\hline & Stage 1 - 2 & 6 & $75.0 \%$ & 2 & $25.0 \%$ & & 6 & $75.0 \%$ & 2 & $25.0 \%$ & \\
\hline $11 \mathrm{NVI}$ & Stage $3-4$ & 2 & $25.0 \%$ & 6 & $75.0 \%$ & 0.017 & 6 & $75.0 \%$ & 2 & $25.0 \%$ & 0.240 \\
\hline & No & 7 & $77.8 \%$ & 2 & $22.2 \%$ & & 6 & $66.7 \%$ & 3 & $33.3 \%$ & \\
\hline Mucinous Histology & Yes & 1 & $14.3 \%$ & 6 & $85.7 \%$ & 0.012 & 6 & $85.7 \%$ & 1 & $14.3 \%$ & 0.383 \\
\hline & Absent & 7 & $53.8 \%$ & 6 & $46.2 \%$ & & 10 & $76.9 \%$ & 3 & $23.1 \%$ & \\
\hline LVI & Present & 1 & $33.3 \%$ & 2 & $66.7 \%$ & 0.522 & 2 & $66.7 \%$ & 1 & $33.3 \%$ & 0.712 \\
\hline & Alive & 8 & $66.7 \%$ & 4 & $33.3 \%$ & & 9 & $75.0 \%$ & 3 & $25.0 \%$ & \\
\hline
\end{tabular}

Tumor location: Proximal refers o right colon and transverse colon; while distal refers to left colon, sigmoid and rectum. Seventh edition if the AJCC/UICC TNM staging system. (American Joint Committee on Cancer. Colon and Rectum. Amin MB, Edge S, Greene F, Byrd DR, Brookland RK, et at, eds. AJCC Cancer staging Manual. $8^{\text {th }}$ ed. New York, NY: Springer; 2017). LVI: Lymphovascular Invasion.

of invasion, and lymph node invasion were not significant predictors for NRAS mutational status.

We also noted that KRAS-mutated carcinomas were more common in the proximal colon. However, this association was not statistically significantly (63.6\% vs $36.4 \%, \mathrm{P}<0.106)$. Besides that, NRAS mutations was found to be associated with distant metastasis but this association did not differ significantly between NRAS-mutated and NRAS-wild type carcinomas. $(\mathrm{p}=0.064)$. 


\subsection{Type and Frequencies of KRAS and NRAS Gene Mutations}

The type of the KRAS and NRAS gene mutations are presented in detail in Table 3. Eight cases were found to harbor at least one mutation in the KRAS gene. On the other hand, three cases with at least one mutation in the NRAS gene were detected. We found one case that had mutations in both KRAS and NRAS genes. For the KRAS gene, the most common mutation in our study group was a missense mutation (87.5\%) then synonymous mutation (12.5\%). While, for the NRAS gene, the most common mutation is missense mutation $(75 \%)$ then stop-gained mutation (25\%).

\subsection{Association of KRAS Polymorphisms with the Clinicopathological Features in Colon Cancer Cases}

To study the association of KRAS polymorphism on colon cancer, we applied Pearson Chi-Square tests with different clinicopathological variables. The number of mutations per case was significantly associated with depth of the invasion $(p=0.049)$. The most common mutation in our study group was a missense mutation. A missense mutation was highly associated with age and gender (both,

Table 3. Distribution of KRAS and NRAS mutation types.

\begin{tabular}{|c|c|c|c|c|c|}
\hline Case Number & Gene & Chromosome & Variant & $\begin{array}{l}\text { Amino Acids } \\
\text { Substitution }\end{array}$ & Consequence \\
\hline \multirow{3}{*}{1} & \multirow{3}{*}{ KRAS } & \multirow{3}{*}{12} & $\mathrm{G}>\mathrm{G} / \mathrm{T}$ & $\mathrm{A} / \mathrm{E}$ & Missense \\
\hline & & & $\mathrm{G}>\mathrm{G} / \mathrm{T}$ & $\mathrm{G}$ & Synonymous \\
\hline & & & $\mathrm{C}>\mathrm{C} / \mathrm{T}$ & $\mathrm{G} / \mathrm{D}$ & Missense \\
\hline \multirow[t]{2}{*}{2} & \multirow[t]{2}{*}{ KRAS } & \multirow[t]{2}{*}{12} & $\mathrm{C}>\mathrm{C} / \mathrm{A}$ & $\mathrm{G} / \mathrm{V}$ & Missense \\
\hline & & & $\mathrm{G}>\mathrm{G} / \mathrm{T}$ & $\mathrm{A} / \mathrm{E}$ & Missense \\
\hline \multirow[t]{4}{*}{3} & \multirow[t]{4}{*}{ KRAS } & \multirow[t]{2}{*}{12} & $\mathrm{C}>\mathrm{C} / \mathrm{G}$ & G/A & Missense \\
\hline & & & $\mathrm{C}>\mathrm{C} / \mathrm{A}$ & $\mathrm{G} / \mathrm{C}$ & Missense \\
\hline & & \multirow{5}{*}{12} & $\mathrm{G}>\mathrm{G} / \mathrm{T}$ & $\mathrm{A} / \mathrm{E}$ & Missense \\
\hline & & & $\mathrm{C}>\mathrm{C} / \mathrm{G}$ & G/A & Missense \\
\hline \multirow[t]{3}{*}{4} & \multirow[t]{3}{*}{ KRAS } & & $\mathrm{G}>\mathrm{G} / \mathrm{T}$ & G & Synonymous \\
\hline & & & $\mathrm{C}>\mathrm{C} / \mathrm{T}$ & $\mathrm{G} / \mathrm{D}$ & Missense \\
\hline & & & $\mathrm{C}>\mathrm{C} / \mathrm{A}$ & $\mathrm{G} / \mathrm{C}$ & Missense \\
\hline \multirow{2}{*}{5} & KRAS & 12 & $\mathrm{C}>\mathrm{C} / \mathrm{A}$ & $\mathrm{G} / \mathrm{V}$ & Missense \\
\hline & NRAS & 1 & $\mathrm{C}>\mathrm{C} / \mathrm{A}$ & $\mathrm{G} /{ }^{*}$ & Stop Gained \\
\hline 6 & KRAS & 12 & $\mathrm{C}>\mathrm{C} / \mathrm{T}$ & $\mathrm{G} / \mathrm{D}$ & Missense \\
\hline 7 & KRAS & 12 & $\mathrm{C}>\mathrm{C} / \mathrm{A}$ & $\mathrm{G} / \mathrm{V}$ & Missense \\
\hline 8 & KRAS & 12 & $\mathrm{C}>\mathrm{C} / \mathrm{A}$ & $\mathrm{G} / \mathrm{V}$ & Missense \\
\hline 9 & NRAS & 1 & $\mathrm{~T}>\mathrm{T} / \mathrm{C}$ & $\mathrm{Q} / \mathrm{R}$ & Missense \\
\hline 10 & NRAS & 1 & $\mathrm{~T}>\mathrm{T} / \mathrm{C}$ & $\mathrm{Q} / \mathrm{R}$ & Missense \\
\hline 11 & NRAS & 1 & $\mathrm{~T}>\mathrm{T} / \mathrm{C}$ & $\mathrm{Q} / \mathrm{R}$ & Missense \\
\hline
\end{tabular}


$\mathrm{p}=0.039)$. In addition, it was highly associated with tumor grade and with mucinous differentiation $(\mathrm{p}=0.014, \mathrm{p}=0.012)$, respectively.

\subsection{Correlation of Mutations with Overall Survival}

The mean follow-up time was $48.9 \pm 16.3$ months (19 - 60 months). KRAS-mutated carcinomas had a significantly worse overall survival $(p=0.025)$ (Figure $1(a)$ ).

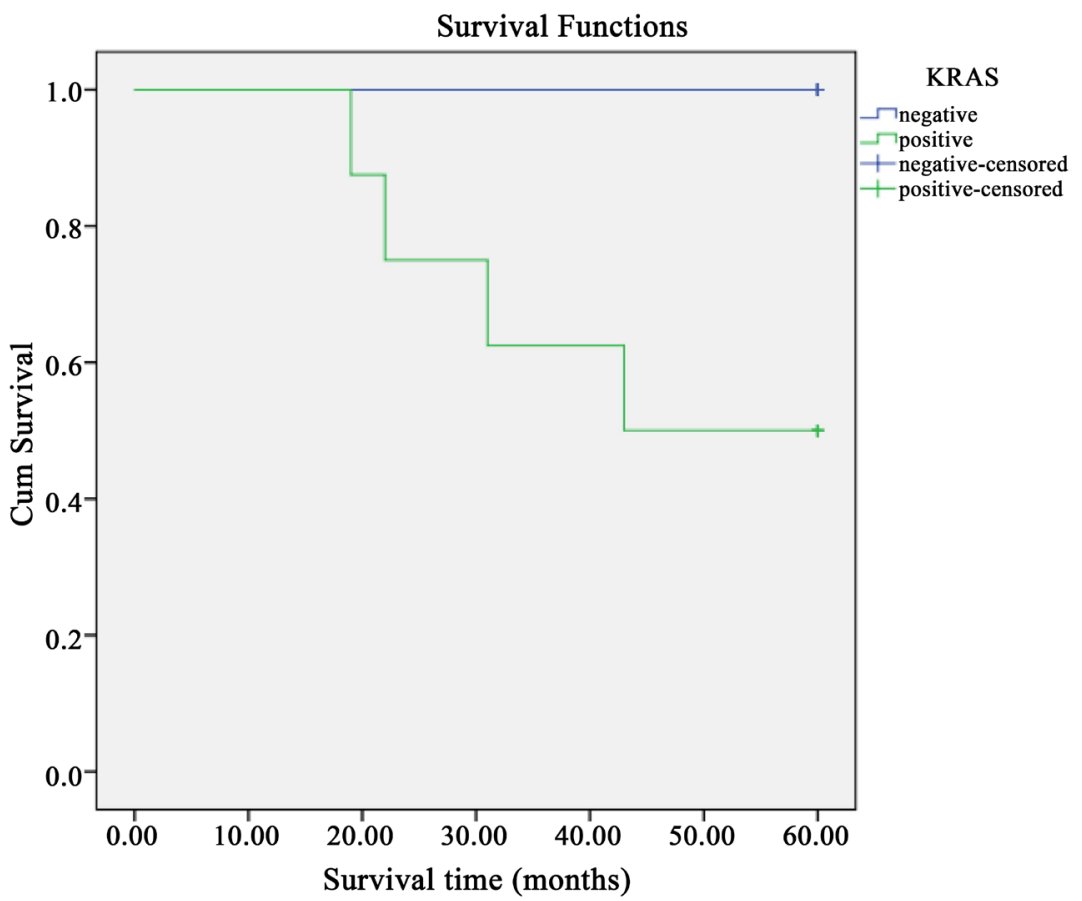

(a)

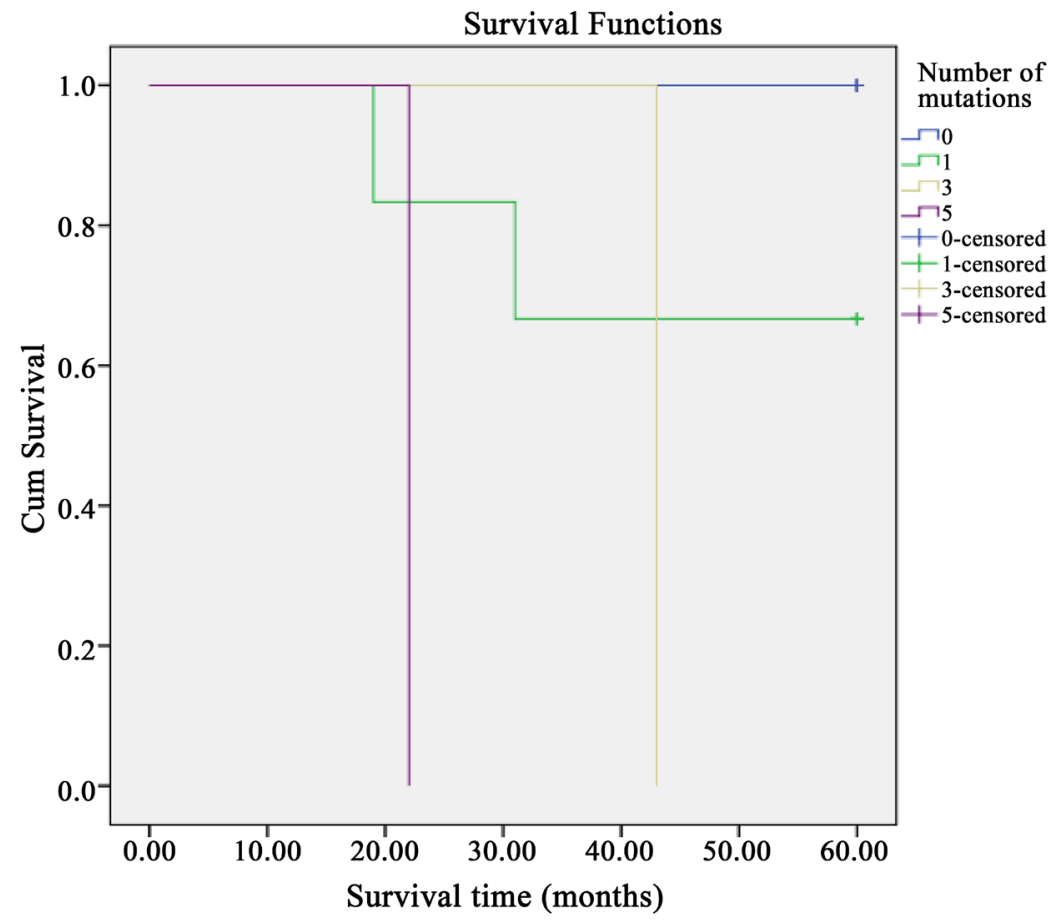

(b) 


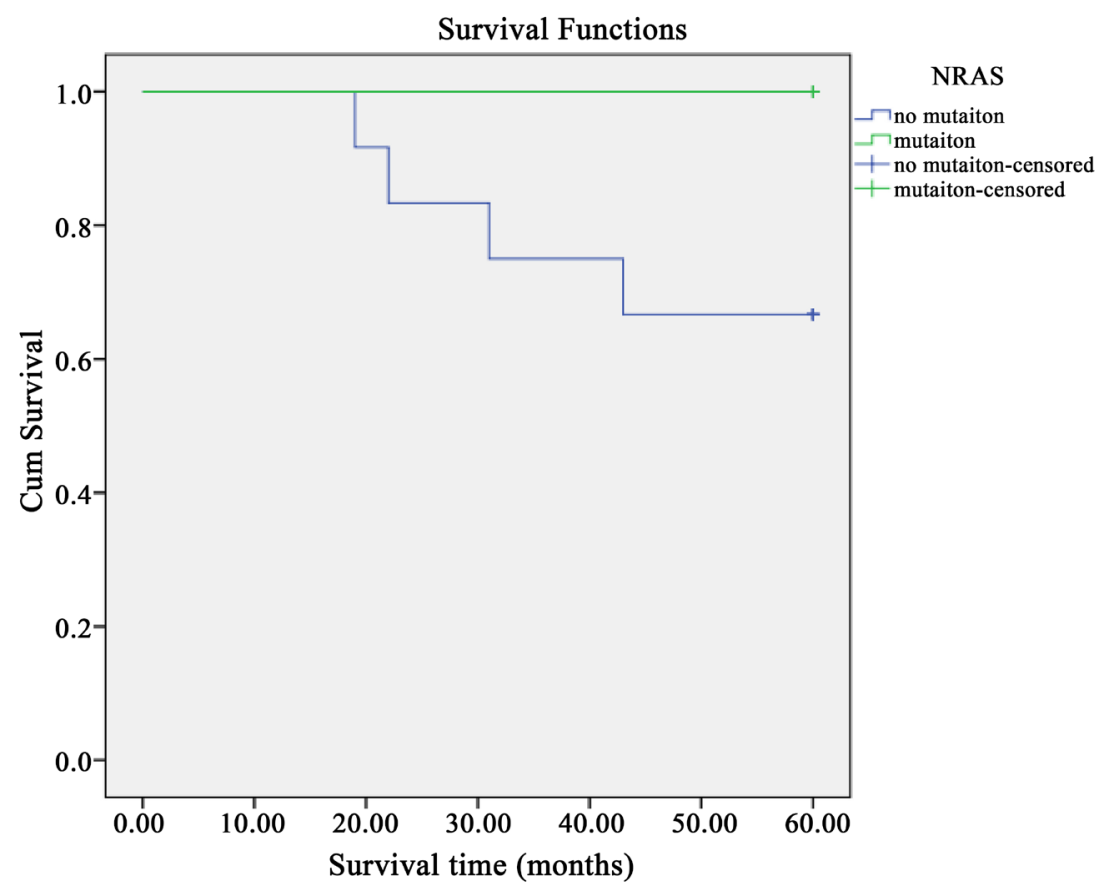

(c)

Figure 1. Kaplan-Meier survival curves for overall survival according to the expression of KRAS (a), (number of KRAS mutations/case) KRAS for different levels of number of mutations, and for (b), and the expression of NRAS (c).

In addition, we found significant correlation between the number of mutations in KRAS in each case and the overall survival. Cases with high number of KRAS mutations had significantly the worst overall survival ( $p<0.0001$ ) (Figure $1(\mathrm{~b})$ ). On the other hand, no significant association has been detected between NRAS mutation and overall survival $(\mathrm{p}=0.985)$ (Figure $1(\mathrm{c})$ ) in our study group.

\section{Discussion}

In this study, we investigated 16 patients with CRC by applying next generation sequencing in the population of the western region of the Saudi Arabia. Due to the main aim of the study, we focused on both genes KRAS and NRAS as prognostic factors. Furthermore, we assessed the association between these KRAS and NRAS mutations with the clinicopathological features which showed significant correlations. It is worth noting that, all the cases were muted for the P53 gene. For the KRAS mutation, the frequency was $50 \%$ of our study group. Several reports, including reports from Saudi, showed that the incidence of KRAS mutation ranged from $28.1 \%$ to $56 \%$ [10] [11] [12] [13]. This is consistent with the frequency of the KRAS mutation in our study [12] [14]. For NRAS mutation, the frequency in our study group was $25 \%$ of patients which was higher comparing the ranges of the previous reports $(2.2 \%$ - 7\%) [14] [15]. Regardless of the small sample size in our study group, these finding are consistent due to the high accuracy of the next generation sequencing.

In this study, we found that KRAS-mutated carcinoma was significantly more 
common in patients who are older than 60 years. Also, it is more common in female patients than the males. These findings are similar to previous studies reported that KRAS mutation was significantly correlated with old age and it is more common in females [16] [17]. On the other hand and contradictory to our results, different studies have shown that KRAS mutations are more common in young patients, less than 50 years old, and the mutation occurrence was comparable between male and female [18].

The correlation between KRAS mutations and tumor location remains debatable. In our study group, we observed that KRAS-mutated carcinomas were more common in right colon than left colon. Numerous studies, similar to our results, have found that tumors exhibiting KRAS mutations are more frequently in the right colon area [11] [19] [20]. Others have shown the KRAS mutation does not correlate with the location of the tumor, while others have reported that KRAS mutation was significantly higher in the left-side of the colon, in disagreement with our study [12] [14].

Comparing the results of CRC patients who were non-mutated in the KRAS gene with patients who had mutations in the KRAS gene, tumors exhibited the later had significantly more mucinous differentiation and significantly associated with higher tumor grade. This finding is consistent with other studies [21] [22]. However, the correlations between KRAS mutation and Clinicopathological characteristics are inconclusive. Some studies found an association between KRAS mutation and tumor grade or stage however others did not [10] [11] [12] [16]. The discrepancy between these studies may be due to lack of clinical and histological data, as well as due to the retrospective observational methodology.

Several reports show similar clinicopathological features between patients with KRAS and NRAS mutation [14] [23]. However, in our results, we could only find that NRAS mutation was more commonly associated with distant metastasis but this association did not differ significantly between NRAS-mutated and non-mutated NRAS patients. Also, we did not find any significant difference regarding age, gender, location of the tumor, mucinous differentiation, or grade between NRAS-mutated and non-mutated patients.

Due to the tumor heterogeneity, we detected 5 patients who had more than 1 mutation, including patients with coexistence of KRAS and either NRAS or PIK3CA genes mutations. This suggests that intratumor heterogeneity does occur in KRAS and NRAS mutant CRC and confirms that CRC is formed in clones with different mutations [15] [24]. In addition, most of these mutations were missense mutations for both KRAS and NRAS genes, except for two synonymous mutations for the KRAS and one stop-gained mutation for the NRAS gene. One interesting case in this study group had a poorly differentiated tumor, mucinous histology, 5 positive lymph nodes and the patient developed lung metastasis, eventually. This case had a high number of genetic variance and was positive for mutations in these genes: NRAS, TP53, EGFR, ERBB2, PDGFRA, KIT, MET and AKT1. This high heterogeneity in this specific case with respect to 
these mutations might have a critical role in worsening the case scenario and it may need implications in considering the therapeutic approaches.

Patient with mutated KRAS and with mutated NRAS respond poorly to anti-EGFR treatment. De Roock et al. analyzed the role of the NRAS mutation in patients treated with Cetuximab and chemotherapy and they found that only one of the 13 patients responded to treatment and similar results were reported by $\mathrm{Au}$ et al. Therefore, and due to the ineffectiveness of anti-EGFR treatment in patients with NRAS mutation, the European Drug Agency does not recommend the use of anti-EGFR drugs in such patients [23] [25].

Concerning prognosis, we found that KRAS-mutated carcinomas had a significantly worse overall survival which is consistent with other published data [13] [20] [26]. On the other hand, we could not detect any correlation between NRAS mutation and overall survival.

The present study is believed to be the first to provide information on frequency and types of KRAS, NRAS, and p53 mutations in Saudi patients with CRC. However, it has some limitations although it is the first to examine the effect of the of KRAS, NRAS and P53 genes mutations on the clinicopathological findings of CRC in Saud population. The small sample size may limit the findings. This is due to the lack of funding. In addition, it is a retrospective research and selection bias inevitably exists, as well as the limited access to the data for prognosis. Given our limited sample size and data, we acknowledge that these observations are speculative. Additional studies are required to understand the correlation of the mutations in these three genes (KRAS, NRAS, and p53) with the development of CRC for better diagnosis and improve the therapeutic effect.

\section{Conflicts of Interest}

The authors declare no conflicts of interest regarding the publication of this paper.

\section{References}

[1] Huyghe, J.R., Harrison, T.A., Bien, S.A., Hampel, H., Figueiredo, J.C., Schmit, S.L., et al. (2021) Genetic Architectures of Proximal and Distal Colorectal Cancer Are Partly Distinct. Gut.

[2] Iacopetta, B. (2002) Are There Two Sides to Colorectal Cancer? International Journal of Cancer, 101, 403-408. https://doi.org/10.1002/ijc.10635

[3] Carethers, J.M. (2011) One Colon Lumen But Two Organs. Gastroenterology, 141, 411-412. https://doi.org/10.1053/j.gastro.2011.06.029

[4] Yamauchi, M., Lochhead, P., Morikawa, T., Huttenhower, C., Chan, A.T., Giovannucci, E., et al. (2012) Colorectal Cancer: A Tale of Two Sides or a Continuum? Gut, 61, 794-797. https://doi.org/10.1136/gutjnl-2012-302014

[5] Arrington, A.K., Heinrich, E.L., Lee, W., Duldulao, M., Patel, S., Sanchez, J., et al. (2012) Prognostic and Predictive Roles of KRAS Mutation in Colorectal Cancer. International Journal of Molecular Sciences, 13, 12153-12168. https://doi.org/10.3390/ijms131012153

[6] Normanno, N., Rachiglio, A.M., Lambiase, M., Martinelli, E., Fenizia, F., Esposito, 
C., et al. (2015) Heterogeneity of KRAS, NRAS, BRAF and PIK3CA Mutations in Metastatic Colorectal Cancer and Potential Effects on Therapy in the CAPRI GOIM Trial. Annals of Oncology, 26, 1710-1714.

[7] Tan, C. and Du, X. (2012) KRAS Mutation Testing in Metastatic Colorectal Cancer. World Journal of Gastroenterology, 18, 5171-5180.

[8] Prior, I.A., Lewis, P.D. and Mattos, C. (2012) A Comprehensive Survey of Ras Mutations in Cancer. Cancer Research, 72, 2457-2467. https://doi.org/10.1158/0008-5472.CAN-11-2612

[9] Sclafani, F., Wilson, S.H., Cunningham, D., Gonzalez De Castro, D., Kalaitzaki, E., Begum, R., et al. (2020) Analysis of KRAS, NRAS, BRAF, PIK3CA and TP53 Mutations in a Large Prospective Series of Locally Advanced Rectal Cancer Patients. International Journal of Cancer, 146, 94-102. https://doi.org/10.1002/ijc.32507

[10] Zekri, J., Al-Shehri, A., Mahrous, M., Al-Rehaily, S., Darwish, T., Bassi, S., et al. (2017) Mutations in Codons 12 and 13 of K-Ras Exon 2 in Colorectal Tumors of Saudi Arabian Patients: Frequency, Clincopathological Associations, and Clinical Outcomes. Genetics and Molecular Research, 16, gmr16019369.

https://doi.org/10.4238/gmr16019369

[11] Mulla, N., Alshareef, A., Syed, A.R. and Al-Jahel, M. (2020) Clinico-Pathological Study of K-Ras Mutations in Colorectal Tumors: A Single-Center Retrospective Study of 51 Patients in Madinah, Saudi Arabia. Cureus, 12, e9978.

https://doi.org/10.7759/cureus.9978

[12] Zahrani, A., Kandil, M., Badar, T., Abdelsalam, M., Al-Faiar, A. and Ismail, A. (2014) Clinico-Pathological Study of K-Ras Mutations in Colorectal Tumors in Saudi Arabia. Tumori, 100, 75-79. https://doi.org/10.1177/1430.15819

[13] Abubaker, J., Bavi, P., Al-Haqawi, W., Sultana, M., Al-Harbi, S., Al-Sanea, N., et al. (2009) Prognostic Significance of Alterations in KRAS Isoforms KRAS-4A/4B and KRAS Mutations in Colorectal Carcinoma. The Journal of Pathology, 219, 435-445. https://doi.org/10.1002/path.2625

[14] Afrăsânie, V.-A., Marinca, M.V., Alexa-Stratulat, T., Gafton, B., Păduraru, M., Adavidoaiei, A.M., et al. (2019) KRAS, NRAS, BRAF, HER2 and Microsatellite Instability in Metastatic Colorectal Cancer-Practical Implications for the Clinician. Radiology and Oncology, 53, 265-274. https://doi.org/10.2478/raon-2019-0033

[15] Normanno, N., Rachiglio, A.M., Lambiase, M., Martinelli, E., Fenizia, F., Esposito, C., et al. (2015) Heterogeneity of KRAS, NRAS, BRAF and PIK3CA Mutations in Metastatic Colorectal Cancer and Potential Effects on Therapy in the CAPRI GOIM Trial. Annals of Oncology, 26, 1710-1714.

[16] Shen, Y., Wang, J., Han, X., Yang, H., Wang, S., Lin, D., et al. (2013) Effectors of Epidermal Growth Factor Receptor Pathway: The Genetic Profiling of KRAS, BRAF, PIK3CA, NRAS Mutations in Colorectal Cancer Characteristics and Personalized Medicine. PLOS ONE, 8, e81628.

https://doi.org/10.1371/journal.pone.0081628

[17] Gao, J., Sun, Z., Li, Y. and Shen, L. (2012) Mutations of KRAS and BRAF in Chinese Patients with Colorectal Carcinoma: Analyses of 966 Cases. Chinese Journal of Pathology, 41, 579-583.

[18] Kafatos, G., Niepel, D., Lowe, K., Jenkins-Anderson, S., Westhead, H., Garawin, T., et al. (2017) RAS Mutation Prevalence among Patients with Metastatic Colorectal Cancer: A Meta-Analysis of Real-World Data. Biomarkers in Medicine, 11, 751-760. https://doi.org/10.2217/bmm-2016-0358

[19] Imamura, Y., Lochhead, P., Yamauchi, M., Kuchiba, A., Qian, Z.R., Liao, X., et al. 
(2014) Analyses of Clinicopathological, Molecular, and Prognostic Associations of KRAS Codon 61 and Codon 146 Mutations in Colorectal Cancer: Cohort Study and Literature Review. Molecular Cancer, 13, 135. https://doi.org/10.1186/1476-4598-13-135

[20] Foltran, L., De Maglio, G., Pella, N., Ermacora, P., Aprile, G., Masiero, E., et al. (2015) Prognostic Role of KRAS, NRAS, BRAF and PIK3CA Mutations in Advanced Colorectal Cancer. Future Oncology (London, England), 11, 629-640. https://doi.org/10.2217/fon.14.279

[21] Lan, Y.-T., Chang, S.-C., Lin, P.-C., Lin, C.-C., Lin, H.H., Huang, S.-C., et al. (2021) Clinicopathological and Molecular Features of Colorectal Cancer Patients with $\mathrm{Mu}$ cinous and Non-Mucinous Adenocarcinoma. Frontiers in Oncology, 11, Article ID: 620146. https://doi.org/10.3389/fonc.2021.620146

[22] Dai, D., Zhou, B., Zhong, Y., Jin, H. and Wang, X. (2019) Survival of Patients with Resected Primary Colorectal Mucinous Adenocarcinoma: A Competing Risk Nomogram Analysis. Oncology Letters, 18, 6594-6604. https://doi.org/10.3892/ol.2019.11024

[23] De Roock, W., De Vriendt, V., Normanno, N., Ciardiello, F. and Tejpar, S. (2011) KRAS, BRAF, PIK3CA, and PTEN Mutations: Implications for Targeted Therapies in Metastatic Colorectal Cancer. The Lancet Oncology, 12, 594-603. https://doi.org/10.1016/S1470-2045(10)70209-6

[24] Sottoriva, A., Kang, H., Ma, Z., Graham, T.A., Salomon, M.P., Zhao, J., et al. (2015) A Big Bang Model of Human Colorectal Tumor Growth. Nature Genetics, 47, 209-216. https://doi.org/10.1038/ng.3214

[25] Au, H.-J., Karapetis, C.S., O’Callaghan, C.J., Tu, D., Moore, M.J., Zalcberg, J.R., et al. (2009) Health-Related Quality of Life in Patients with Advanced Colorectal Cancer Treated with Cetuximab: Overall and KRAS-Specific Results of the NCIC CTG and AGITG CO.17 Trial. Journal of Clinical Oncology, 27, 1822-1828.

[26] Garcia-Carbonero, N., Martinez-Useros, J., Li, W., Orta, A., Perez, N., Carames, C., et al. (2020) KRAS and BRAF Mutations as Prognostic and Predictive Biomarkers for Standard Chemotherapy Response in Metastatic Colorectal Cancer: A Single Institutional Study. Cells, 9, 219. https://doi.org/10.3390/cells9010219 